\title{
@ \\ Microscopic picture of the critical state in a superconductor with a periodic array of antidots
}

\author{
A. V. Silhanek, J. Gutierrez, R. B. G. Kramer, G. W. Ataklti, J. Van de Vondel, and V. V. Moshchalkov \\ INPAC-Institute for Nanoscale Physics and Chemistry, Nanoscale Superconductivity and Magnetism Group, K.U. Leuven, \\ Celestijnenlaan 200D, B-3001 Leuven, Belgium \\ A. Sanchez \\ Departament de Fisica, Universitat Autonoma de Barcelona, 08193 Bellaterra, Barcelona, Spain
}

(Received 19 November 2010; published 28 January 2011)

\begin{abstract}
By using scanning Hall microscopy we visualize the progressive formation of the critical state with individual vortex resolution in a $\mathrm{Pb}$ thin film with a periodic pinning array. Slightly above the first penetration field, we directly observe a terraced critical state as proposed theoretically by Cooley and Grishin [Phys. Rev. Lett. 74, 2788 (1995)]. However, at higher fields, the flux front tends to disorder and the classical Bean profile is restored. This study allows us to establish a clear link between the widely used integrated response measurements in the superconducting state and the nanoscale landscape defined by individual vortices.
\end{abstract}

DOI: 10.1103/PhysRevB.83.024509

PACS number(s): 74.78.-w, 74.25.Ha, 74.25.Uv, 74.25.Wx

\section{INTRODUCTION}

In 1962 Bean demonstrated that the magnetic response of type-II superconductors arises from the irreversible process of flux penetration-flux exit at the sample borders. ${ }^{1}$ This irreversibility results, in turn, from the different metastable states determined by the ubiquitous vortex pinning centers. In his original work, Bean ignored edge barriers and assumed an homogeneous and constant critical current. This simplified model has been later on extended to include effects of screening currents for fields close to the lower critical field, ${ }^{2}$ demagnetization and self-field effects, ${ }^{3}$ nonlocal relations between the local vortex density and the magnetic induction, ${ }^{4}$ as well as more realistic field-dependent critical currents. ${ }^{5}$

In spite of the apparent oversimplification of the Bean model, the continuous success of its application to quantitatively estimate the critical current in all sort of superconducting materials evidences its ability to capture the essential physical ingredients. However, because this model relies on a macroscopic description of the internal field profile that disregards individual vortices, it has been so far little scrutinized at the single-vortex resolution level. ${ }^{6-8}$ This limit is particularly relevant in the case of fields just above the lower critical field or for ac-susceptibility measurements of small field amplitude such that few vortices penetrate into the sample.

In this work we present local magnetic-field measurements via scanning Hall probe microscopy (SHPM) on a thin superconducting sample containing a periodic array of antidots and for external fields applied perpendicularly to the plane of the film. The large critical current produced by the antidot array ensures that measurements performed in field-cooling (FC) conditions are very close to the stable vortex patterns. ${ }^{2}$ Additionally, zero-field-cooling (ZFC) measurements were carried out in order to investigate the progressive formation of the critical state. We found that for fields just above the penetration field, $H^{*}$, a stepwise flux gradient develops as a consequence of the periodic pinning landscape. This represents the first direct visualization of the so-called "terraced critical state" introduced in Ref. 9. At higher fields, the flux front tends to disorder. Interestingly, by slowly modulating the external field it is possible to determine the ac response of the sample from the snapshots of the vortex distribution.

\section{EXPERIMENTAL DETAILS}

The investigated sample consists of a $\mathrm{Pb}$ film of thickness $t=50 \mathrm{~nm}$ with a triangular array of antidots obtained by electron-beam evaporation and subsequent lift-off. The sample was deposited on top of a $\mathrm{SiO}_{2}$ insulating substrate. The antidots have a V shape with each leg of the $\mathrm{V}$ being $0.8 \mu \mathrm{m}$ long and $0.2 \mu \mathrm{m}$ wide and forming an angle of $120^{\circ}$ [see Fig. 1(a)]. The period of the pattern is $w=3 \mu \mathrm{m}$, which corresponds to a commensurability field $H_{1}=\frac{2 \Phi_{0}}{\sqrt{3} w^{2}} \sim 2.7 \mathrm{G}$ at which the density of vortices and antidots coincide, as shown in Fig. 1(c). Typical values of superconducting coherence length and penetration depth at zero temperature estimated in similar samples are $\xi(0) \sim 34 \mathrm{~nm}$ and $\lambda(0) \sim 50 \mathrm{~nm}$, respectively. The superconductor-to-normal transition at zero field occurs at $T_{c}=7.2 \mathrm{~K}$. More details concerning the sample preparation can be found in Ref. 10.

The SHPM images were obtained by using a modified low-temperature scanning Hall probe microscope from Nanomagnetics Instruments. As schematically shown by the dashed box in Fig. 1(a), the scanning area is $12 \times 12 \mu \mathrm{m}^{2}$. The Hall sensor lies $\sim 500 \mathrm{~nm}$ above the surface of the sample, giving rise to a maximum signal of $\sim 2.5 \mathrm{G}$ at the core of a single quantized vortex, whereas the field resolution of the Hall cross is better than $0.1 \mathrm{G}$.

\section{EQUILIBRIUM VORTEX DISTRIBUTION}

Equilibrium vortex configurations for a superconducting sample with a triangular array of pinning sites has been theoretically studied via molecular dynamics simulation in Refs. 11 and 12. Stable vortex arrangements are expected for every field at which the vortex lattice commensurates with the antidot lattice. Experimentally we can obtain near-to-equilibrium vortex distributions by performing FC experiments. ${ }^{2}$ Some examples of the resulting flux patterns directly visualized via SHPM at $T=6.9 \mathrm{~K}$ and for different applied fields are 
(a)
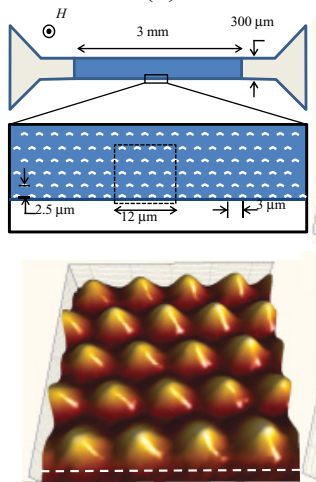

(c) $H=H_{1}$

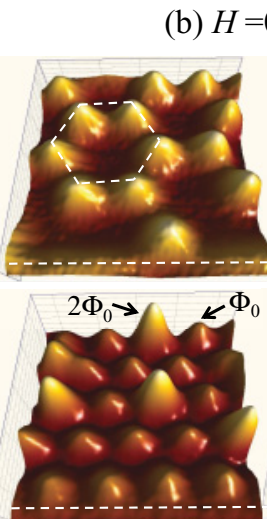

(d) $H=1.3 H_{1}$ (b) $H=0.63 H_{1}$

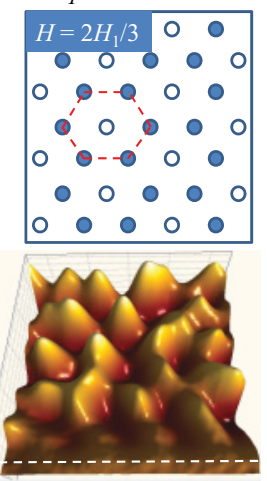

(e) $H=2 H_{1}$

FIG. 1. (Color online) (a) Layout of the investigated sample. The upper panel shows the transport bridge with a 3-mm-long patterned area. The lower panel shows a zoom-in close to the sample's border. The box delimited by the dashed line indicates the area where most of the scanning Hall microscopy images were obtained. (b)-(e) show scanning Hall probe microscopy images obtained at the sample's border, as indicated by the dashed box in (a) after cooling down to $T=6.9 \mathrm{~K}$ in presence of a field (b) $H=0.63 H_{1}$, (c) $H=H_{1}$, (d) $H=1.3 H_{1}$, and (e) $H=2 H_{1}$. The image in (b) is accompanied by the theoretically predicted vortex distribution (Ref. 12). The white dashed line indicates the sample's border. The field picked up by the Hall cross on top of a single quantum vortex is $\sim 2.5 \mathrm{G}$.

shown in Fig. 1. Here the white dashed line indicates the edge of the sample. Figure 1(b) shows a regular honeycomb array of vortices obtained at $H=1.7 \mathrm{Oe} \sim 2 / 3 H_{1}$. Additional submatching regular patterns have been identified at $H=$ $0.5 H_{1}$ and $H=0.25 H_{1}$. These submatching fields are in agreement with previous theoretical predictions. ${ }^{12}$ Figure 1(c) corresponds to the first matching field, where every antidot is occupied with a single vortex. For fields above the first matching field [see Fig. 1(d)] most of the extra vortices $\left(\sim H-H_{1}\right)$ lead to double occupancy $\left(2 \Phi_{0}\right)$ of some pinning sites instead of sitting at interstitial positions. ${ }^{13-15}$ Owing to the repulsive vortex-vortex interaction, double-quantized vortices avoid being located at neighboring holes. Interestingly, doublequantized vortices also avoid sitting at the row of antidots adjacent to the sample's border. This effect results from the fact that this row of antidots is only $0.2 \mu \mathrm{m}$ away from the sample's border, a distance that is smaller than the size of a vortex at this temperature $\left(\sim \Lambda=\lambda^{2} / t \approx 1.7 \mu \mathrm{m}\right)$. Therefore, vortices sitting at this particular row experience a substantial force toward the sample border. This effect is also responsible for a vortex-free band next to the sample border in ZFC experiments as reported in Ref. 8 via magneto-optic experiments. At $H=2 H_{1}$ [Fig. 1(e)] the presence of interconnected local-field peaks evidences the existence of interstitial vortices. Closer to $T_{c}$, double occupancy becomes unfavorable and the local-field landscape shows the presence of interstitial vortices coexisting with a single-vortex occupancy. The mere existence of regular patterns in the above FC experiments is indicative of a low dispersion in pinning energy among different pinning sites and a highly homogeneous sample. ${ }^{13-15}$

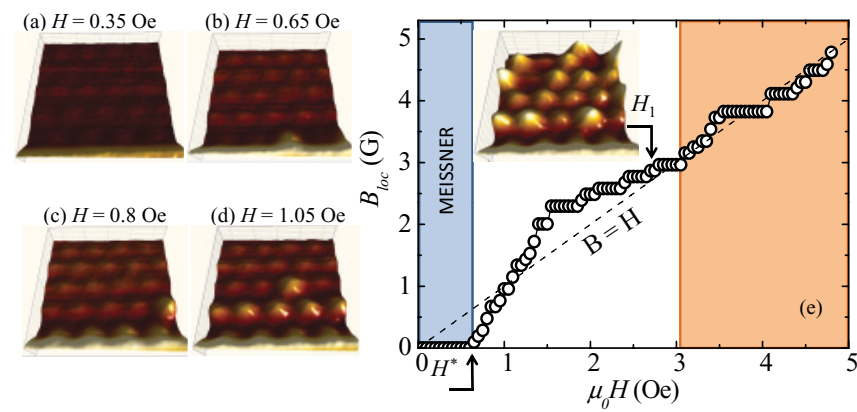

FIG. 2. (Color online) SHPM images obtained at the sample's border as indicated by the dashed box in Fig. 1 after ZFC at $T=6.9 \mathrm{~K}$ for a field (a) $H=0.35 \mathrm{Oe}$, (b) $H=0.65 \mathrm{Oe}$, (c) $H=0.8 \mathrm{Oe}$, (d) $H=1.05 \mathrm{Oe}$, and [inset of (e)] $H=H_{1}=2.7$ Oe. (e) shows the local field $B_{\text {loc }}$ corresponding to the density of vortices in the scanning area as a function of the external field at $T=6.9 \mathrm{~K}$.

\section{FLUX PENETRATION}

Let us now switch to the less explored physics of the flux penetration under ZFC conditions. Figure 2 shows SHPM images at the same spot as in Fig. 1, next to the sample's edge, at $T=6.9 \mathrm{~K}$ and after a progressive increase of the external field. At low fields [Fig. 2(a)] no vortices are observed inside the sample, corresponding to the Meissner state. The strong screening currents circulating close to the border of the sample give rise to the well-known magnification of the total field close to the sample's edge, as clearly evidenced by the bright rim at the bottom of all SHPM images shown in Fig. 2. Increasing the field in steps of 0.05 Oe it is possible to determine the field $H^{*}$ at which the first vortex appears in the scanned area, as shown in Fig. 2(b). For fields slightly above $H^{*}$ the first row (i.e., the closest to the sample's border) of antidots is filled before a vortex appears in the second row [Fig. 2(c)]. Further increasing the external field leads to a completion of the second row of antidots, as shown in Fig. 2(d). These findings represent, to the best of our knowledge, the first experimental confirmation of the terraced critical state where the field front penetration separates a region of local field $B=0$ from another region with constant field $B=H_{1}$, as earlier predicted by Cooley and Grishin. ${ }^{9}$ It is important to notice that this behavior, i.e., the progressive filling of rows of antidots, persists only until the second row is filled. Beyond this row (or field) a far more complex penetration is observed. Indeed, (i) vortices can move further inside the sample, skipping empty rows of antidots, and (ii) increasing field leads to the appearance of double-quantized vortices, although they never nucleate at the sample's border as shown in the inset of Fig. 2(e). As already pointed out above, the latter effect can be attributed to the small separation between the border of the sample and the first row of antidots. In other words, the one-dimensional (1D) model proposed in Ref. 9 is valid only in a narrow field window above $H^{*}$. The richer penetration dynamics observed at higher fields has been partially accounted by the extended version of the terraced field profile to more realistic two-dimensional (2D) periodic arrays done by Reichhardt et al. ${ }^{16}$ using molecular-dynamics simulations assuming a maximum trapping of one vortex per pinning site. 
It is interesting to compare the contrasting vortex distribution obtained at $T=6.9 \mathrm{~K}$ and $H=H_{1}$ after FC [Fig. 1(c)] with that obtained under ZFC conditions [inset of Fig. 2(e)]. Clearly, the very stable periodic arrangement obtained after FC, which typically manifests itself as a local enhancement of the critical current, is not reproducible after ZFC. This difference between FC and ZFC is particularly prominent when $H_{1}$ lies below the field necessary to reach complete field penetration, $H_{p} .{ }^{17}$ Because $H_{p}$ increases as $T$ decreases, this situation is experimentally achieved at temperatures not very close to the critical temperature, and this explains the lack of matching features in global magnetization measurements at low $T .^{18,19}$ In Ref. 16 the authors considered the case that $H_{p}<H_{1}$, and show that local enhancement of the magnetization occurs slightly above the exact matching field as a result of the flux gradient.

As we mentioned above, the images shown in Figs. 2(a)2(e) represent a selected set among a more detailed series with field progressively increasing in uniform field steps of $0.05 \mathrm{Oe}$. From these images it is possible to estimate a local field $B_{\mathrm{loc}}=$ $N \Phi_{0} / A$, where $N$ is the number of flux quanta in the scanning area $A$. Figure 2(e) shows the obtained $B_{\mathrm{loc}}$ as a function of the applied field $H$ for $T=6.9 \mathrm{~K}$. Other measurements performed at 7 and $7.1 \mathrm{~K}$ reveal similar behavior. Because by definition $B_{\text {loc }}$ ignores the field generated by the screening currents, the Meissner phase is characterized by a region where $B_{\mathrm{loc}}=0$. Beyond $H=H^{*}, B_{\text {loc }}$ increases monotonically with a steeper slope than the $\mu_{0} H$ curve indicated by a dotted line. The fact that for a certain field region, $B_{\text {loc }}>\mu_{0} H$ results from the magnification of the local field, i.e., the compression of flux lines next to the sample border, caused by demagnetization effects. Eventually, at high fields $B_{\text {loc }}$ increases linearly with $\mu_{0} H$, as expected for thin-film geometry in a perpendicular magnetic field. ${ }^{20}$ It is worth noticing that at $H=H_{1}$ there is no particular feature (such as a wider plateau of constant $B_{\mathrm{loc}}$ ), indicating a more stable vortex configuration, in agreement with the disordered flux pattern shown in the inset of Fig. 2(e).

\section{MINOR HYSTERESIS LOOPS AND AC SUSCEPTIBILITY}

The graded flux penetration in a superconductor under ZFC conditions gives rise to an irreversible magnetization typically much larger than the equilibrium magnetization, as illustrated by the difference between Figs. 1(c) and 2(e). A very popular way to investigate this irreversible behavior consists of analyzing the magnetic response under small ac-field excitations, i.e., the ac-susceptibility technique. ${ }^{17,21}$ This experimental method has been intensively used in the past to study superconductors with a periodic array of traps. ${ }^{22}$ Most of this research has been done in conventional (low $T_{c}$ ) superconductors where the ac response is weakly dependent on the excitation frequency (up to $10 \mathrm{kHz}$ ), indicating that vortex relaxation processes are not relevant. ${ }^{23}$ This frequencyindependent ac response allows us to directly visualize the vortex response under very slowly $(\sim 2 \mathrm{mHz})$ varying fields. The results of these measurements, obtained at $T=7 \mathrm{~K}$ and with an ac amplitude of $1 \mathrm{Oe}>H^{*}$, are summarized in Fig. 3. Figure 3(a) shows the time variation of the applied magnetic field (red dots) together with the calculated $B_{\text {loc }}$ local induction
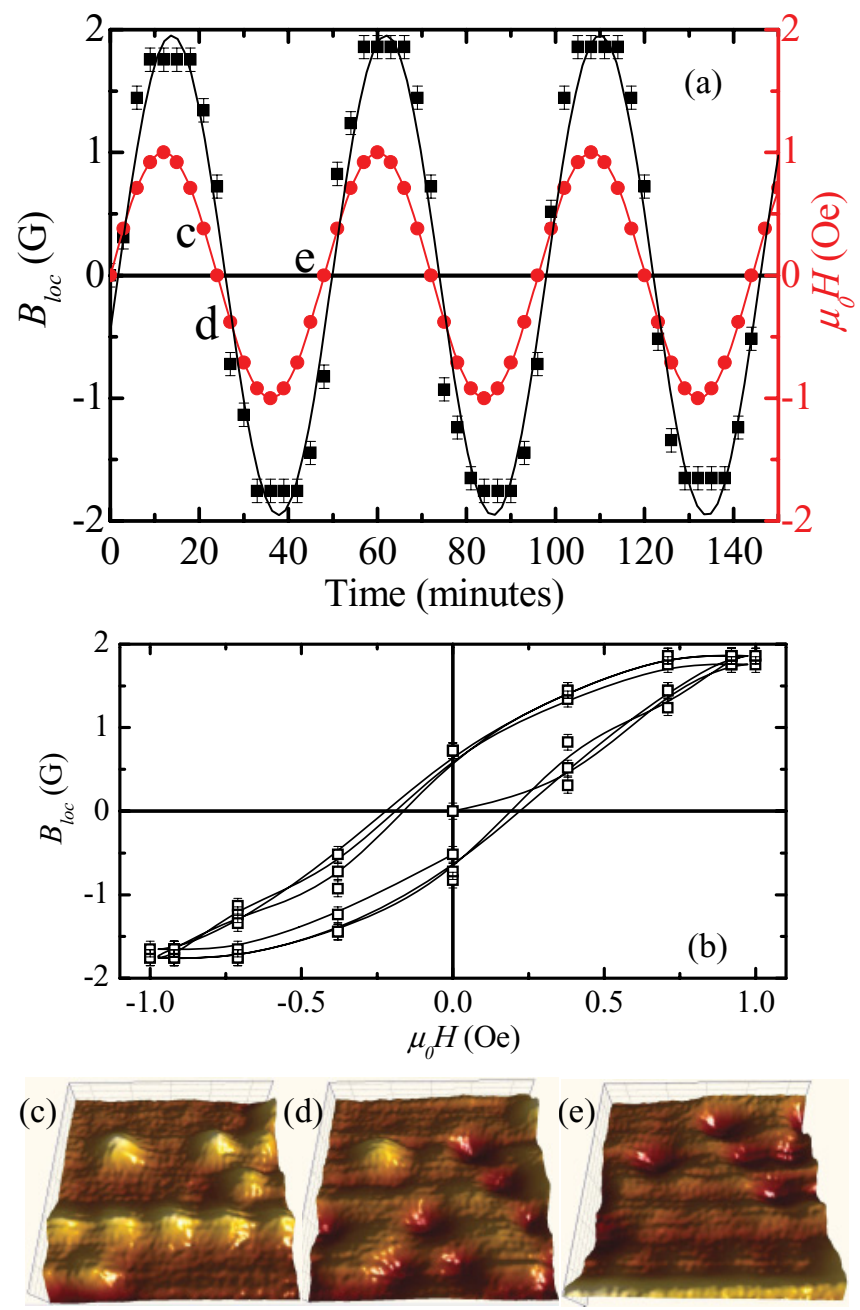

FIG. 3. (Color online) (a) External periodic field $H$ and locally averaged field $B_{\mathrm{loc}}$ as a function of time. (b) Same data as in (a), showing $B_{\text {loc }}$ as a function of $H$. (c)-(e) show SHPM images of the vortex distribution for $T=7 \mathrm{~K}$ obtained at the border of the sample for $H=+0.3 \mathrm{Oe}, H=0.0 \mathrm{Oe}$, and $H=-0.3 \mathrm{Oe}$, respectively. The field picked up by the Hall cross on top of a positive single-quantum vortex is $\sim+2.5 \mathrm{G}$, whereas the field on top of a single quantized antivortex is $\sim-2.5 \mathrm{G}$.

obtained from the SHPM images (black solid squares). Two main features can be observed in the $B_{\text {loc }}$-versus-time curve. First, the amplitude of oscillation of $B_{\mathrm{loc}}$ is larger than that of $H$, as a result of the demagnetization effects. Second, $B_{\text {loc }}$ oscillates out of phase with respect to $H$, indicative of an irreversible behavior. Within a first-order approximation, this irreversibility is accounted for by the relative weight of the ac-susceptibility first-harmonic coefficients $\chi^{\prime}$ and $\chi^{\prime \prime}$, such that $B_{\mathrm{loc}}(t)=B_{0}\left[\chi^{\prime} \cos (\omega t)+\chi^{\prime \prime} \sin (\omega t)\right]$, where $\omega$ is the frequency of oscillation of the alternating field. The solid black line in Fig. 3(a) is a fit to the experimental $B_{\mathrm{loc}}(t)$ data, assuming $\chi^{\prime \prime} / \chi^{\prime}=0.24$. Naturally, this rough estimation is not able to reproduce the flat tops of the $B_{\mathrm{loc}}(t)$ curve, for which not only higher-order harmonics would be necessary but also to perform a more accurate $B_{\text {loc }}$ estimation by increasing the scanning area. This irreversible behavior, characteristic of Bean penetration, is better illustrated in Fig. 3(b), where the minor loop $B_{\text {loc }}(H)$ obtained from Fig. 3(a) has been plotted. 
The initial increase of the external ac field leads to a progressive vortex penetration, as already described above. Once the external field starts to decrease [see Fig. 3(c)] the row of antidots next to the samples' edge is the first to be empty. Upon reversing the polarity of the applied field [see Fig. 3(d)], a coexistence of vortices and antivortices is observed. At the nodes of the ac excitation where $H(t)=0$, i.e., at remanence, a trapped magnetic flux is detected as shown in Fig. 3(e).

\section{CONCLUSION}

In summary, we present direct imaging of the vortex penetration in a superconducting thin film with nanoengineered pinning centers in perpendicular field geometry. Unlike previous investigations, ${ }^{7}$ where the field penetrates into a patterned area but without a physical border, now we address the effects of the sample's border. We observe that the flux front first penetrates, forming a terraced critical state, as theoretically anticipated in Ref. 9 and suggested by integrated response measurements, ${ }^{24}$ but it turns toward a more disordered penetration as the external field increases. By driving the system with a low-frequency acfield excitation, we are able to "visualize," with unprecedented resolution, the dephasing between the drive and the ac response of the vortex lattice. This investigation also demonstrates that the lack of matching features at low temperatures in periodic pinning arrays is a consequence of the self-fields rather than a competition with random point defects.

\section{ACKNOWLEDGMENTS}

This work was supported by Methusalem Funding by the Flemish Government, the FWO-Vlaanderen, and the Belgian Inter-University Attraction Poles IAP Programmes. J.G. is grateful for the financial support of the Spanish Ministerio de Ciencia e Innovacion. A.V.S. and J.V.d.V. are grateful for the support from the FWO-Vlaanderen. Financial support given by Consolider Project NANOSELECT (CSD2007-00041) is acknowledged.
${ }^{1}$ C. P. Bean, Rev. Mod. Phys. 36, 31 (1964).

${ }^{2}$ J. R. Clem and Z. Hao, Phys. Rev. B 48, 13774 (1993).

${ }^{3}$ M. Däumling and D. C. Larbalestier, Phys. Rev. B 40, 9350 (1989).

${ }^{4}$ L. M. Fisher et al., Physica C 245, 231 (1995).

${ }^{5}$ Y. B. Kim, C. F. Hempstead, and A. R. Strnad, Rev. Mod. Phys. 36, 43 (1964).

${ }^{6}$ M. Marchevsky, L. A. Gurevich, P. H. Kes, and J. Aarts, Phys. Rev. Lett. 75, 2400 (1995).

${ }^{7}$ K. Harada, O. Kamimura, H. Kasai, T. Matsuda, A. Tonomura, and V. V. Moshchalkov, Science 274, 1167 (1996).

${ }^{8}$ A. A. F. Olsen, H. Hauglin, T. H. Johansen, P. E. Goa, and D. Shantsev, Physica C 408-410, 537 (2004).

${ }^{9}$ L. D. Cooley and A. M. Grishin, Phys. Rev. Lett. 74, 2788 (1995).

${ }^{10}$ S. Raedts, A. V. Silhanek, V. V. Moshchalkov, J. Moonens, and L. H. A. Leunissen, Phys. Rev. B 73, 174514 (2006).

${ }^{11}$ C. Reichhardt, C. J. Olson, and F. Nori, Phys. Rev. B 57, 7937 (1998).

${ }^{12}$ C. Reichhardt and N. Gronbech-Jensen, Phys. Rev. B 63, 054510 (2001).

${ }^{13}$ A. N. Grigorenko et al., Phys. Rev. B 63, 052504 (2001).

${ }^{14}$ S. B. Field, S. S. James, J. Barentine, V. Metlushko, G. Crabtree, H. Shtrikman, B. Ilic, and S. R. J. Brueck, Phys. Rev. Lett. 88, 067003 (2002).

${ }^{15}$ A. N. Grigorenko, S. J. Bending, M. J. Van Bael, M. Lange, V. V. Moshchalkov, H. Fangohr, P. A. J. de Groot, Phys. Rev. Lett. 90, 237001 (2003).
${ }^{16}$ C. Reichhardt, J. Groth, C. J. Olson, Stuart B. Field, and Franco Nori, Phys. Rev. B 54, 16108 (1996).

${ }^{17}$ J. R. Clem and A. Sanchez, Phys. Rev. B 50, 9355 (1994).

${ }^{18}$ See, for instance, Fig. 4 in A. V. Silhanek, S. Raedts, and V. V. Moshchalkov, Phys. Rev. B 70, 144504 (2004).

${ }^{19}$ A. A. Zhukov et al., Physica C 404, 455 (2004).

${ }^{20}$ A. Sanchez and C. Navau, Supercond. Sci. Technol. 14, 444 (2001).

${ }^{21}$ A. M. Campbell and J. E. Evetts, Adv. Phys. 21, 199 (1972); C. J. van der Beek, V. B. Geshkenbein, and V. M. Vinokur, Phys. Rev. B 48, 3393 (1993); E. H. Brandt, Phys. Rev. B 49, 9024 (1994); F. Gömory, Supercond. Sci. Technol. 10, 523 (1997).

${ }^{22}$ V. V. Metlushko, L. E. DeLong, M. Baert, E. Rosseel, M. J. Van Bael, K. Temst, V. V. Moshchalkov, and Y. Bruynseraede, Europhys. Lett. 41, 333 (1998); V. Metlushko, U. Welp, G. W. Crabtree, Zhao Zhang, S. R. J. Brueck, B. Watkins, L. E. DeLong, B. Ilic, K. Chung, and P. J. Hesketh, Phys. Rev. B 59, 603 (1999); A. V. Silhanek, S. Raedts, M. Lange, and V. V. Moshchalkov, ibid. 67, 064502 (2003); S. Raedts, A. V. Silhanek, M. J. Van Bael, and V. V. Moshchalkov, ibid. 70, 024509 (2004).

${ }^{23}$ A. V. Silhanek, S. Raedts, M. J. Van Bael, and V. V. Moshchalkov, Eur. Phys. J. B 37, 19 (2004).

${ }^{24}$ A. Terentiev, D. B. Watkins, L. E. DeLong, L. D. Cooley, D. J. Morgan, and J. B. Ketterson, Phys. Rev. B 61, R9249 (2000). 